\title{
On-site focus alignment of a fresnel mirror field ${ }^{\text {is }}$
}

\author{
Alois Resch ${ }^{*}$ (D) and Thomas Aigenbauer \\ University of Applied Sciences Upper Austria, Energy Research Group ASIC, 4600 Wels, Austria
}

Received: 29 June 2021 / Received in final form: 15 July 2021 / Accepted: 15 July 2021

\begin{abstract}
Linear concentrating Fresnel collectors are available in various scales for providing solar thermal heat in the temperature range beyond $100^{\circ} \mathrm{C}$. For raising the flexibility in applying such collector systems on mounting sites with restricted space availability, the presented research project investigated the possibilities for adapting an existing Fresnel collector with a mirror surface of $22 \mathrm{~m}^{2}$ towards more compactness by using only one half of the mirror field. Therefore, the receiver was relocated and the focus alignment of the mirrors had to be adapted. The theoretical approach by geometrical calculation of the single mirror angles using MATLAB ${ }^{\mathrm{TM}} \mathrm{was}$ successful, although the required effort for developing such an optical model was high. Furthermore, the resulting angle information obtained by the calculation is difficult to realise on the mirror field, because a highprecision goniometer would be necessary in order to adjust the mirror stripes accordingly. On the other hand, an experimental approach was analysed for realising on-site focus alignment of the single mirror field. The focussed beam of a conventional laser pointer was directed to each of the mirror stripes, while adjusting the corresponding mirror angle until the reflected beam impinged on the center of the receiver. In the end, the experimental procedure turned out to be favourable, because it is simple and reliable, and it requires much less effort. In this way, the adaption of the Fresnel mirror field was successful, as the concentration and the tracking is working as expected.
\end{abstract}

\section{Introduction}

The transition of the world́s energy supply system towards an emission-free future can be seen as the central challenge of this century. Within the last years, this topic has been attracting more and more public attention, and indeed it seems like the change has really started. Electric mobility is currently launching all over the world, and the generation of renewable electricity by photovoltaics, wind or hydropower stations reveals a significant annual boost. In 2019, an additional power capacity of more than $200 \mathrm{GW}_{\mathrm{el}}$ of emission-free electricity production was globally installed [1], confirming that the world́s community is heading for the right direction. However, beside these very positive developments in terms of renewable electricity production, it has to be pointed out that the share of electrical energy demand on the total final energy consumption was $17 \%$ in 2017. Even if the entire electricity production could be converted into renewable forms, the energy transition

\footnotetext{
World Renewable Energy Conference, Lisbon 2020, 13-18 September 2020 - IST Lisboa.

* e-mail: alois.resch@fh-wels.at
}

for the transport sector (32\% share of final energy consumption) and the heating and cooling sector, accounting for $51 \%$ of the global energy demand, would still have to be solved [1].

Focussing on the heating and cooling sector, $39 \%$ of this thermal energy demand is taken from the industry, both for space heating or cooling and for process heating or cooling [2]. In 2019, solar thermal systems with an installed power of $479 \mathrm{GW}_{\text {th }}$ were in operation worldwide, but only $2 \%$ of this huge renewable thermal power is applied to industrial purposes (including solar district heating and solar cooling) [3]. One reason for this discrepancy between the massive heat demand in the industry and the low contribution of solar thermal systems to feed this consumption is the temperature requirement of industrial heat sinks. $72 \%$ of the heat demand in the industry requests temperatures above $100{ }^{\circ} \mathrm{C}$ [4], where conventional solar thermal collector systems already reveal reduced efficiency $[5,6]$.

By contrast, concentrating solar thermal collectors represent a possibility to supply renewable heat for industrial processes with improved efficiency, especially in temperature ranges beyond $100^{\circ} \mathrm{C}$. In this regard, linear Fresnel mirror fields are well established, and collector systems of various scales are available [7-9]. 


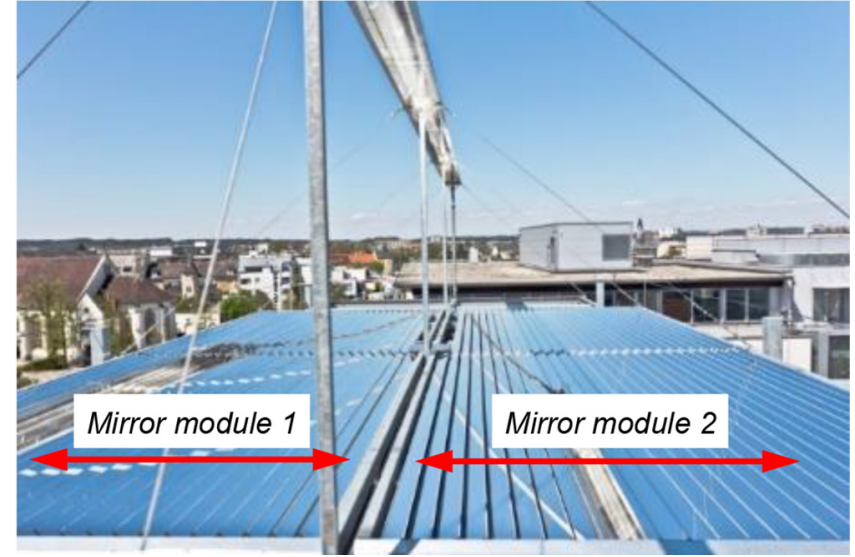

Fig. 1. Fresnel collector on rooftop of FHOOE.

\section{Methodology}

The University of Applied Sciences Upper Austria (FHOOE) operates a Fresnel collector from the Austrian supplier FRESNEX with a total mirror area of $22 \mathrm{~m}^{2}$ consisting of two halves resp. two mirror modules. The receiver is centered above the two halves and the mirrors of both modules are aligned accordingly, see Figure 1.

The mirror stripes of each module are mechanically coupled by a control rod, connected to a servo motor. Based on location and time, the control software calculates the position of the Sun and drives the two motors accordingly.

If only one mirror module with $11 \mathrm{~m}^{2}$ of surface could be used, e.g. due to space limitations on a mounting site in urban context, the receiver has to be re-centered above the single module. Hence, the focus alignment of the mirrors is not suitable anymore. In order to gain more flexibility in applying the considered Fresnel collector, the project work described in this paper was carried out with the main aim to find a practial way for aligning the focus from a single mirror module, directly on the collector mounting site.

The following sub-sections describe details in the mechanical construction of the Fresnel collector in order to illustrate how the focus alignment can be implemented. Furthermore, a theoretical and an experimental method for finding the required mirror positions is presented.

\subsection{Mechanical adjustment of mirror stripe angles}

Generally, in a linear Fresnel collector system, each mirror stripe requires a specific angle between the mirror plane and the horizontal plane, so that each reflected beam can hit the receiver. With every change of the Sun position, the mirror angles have to be adapted as well. As this required stepwise adaption of the mirror angles is the same in absolute numbers for all mirror stripes, the relative angles between the separate stripes remain constant. Therefore, the single mirrors can be mechanically connected, so that the tracking of the Sun position can be done with one central control rod, driven by only one servo motor.

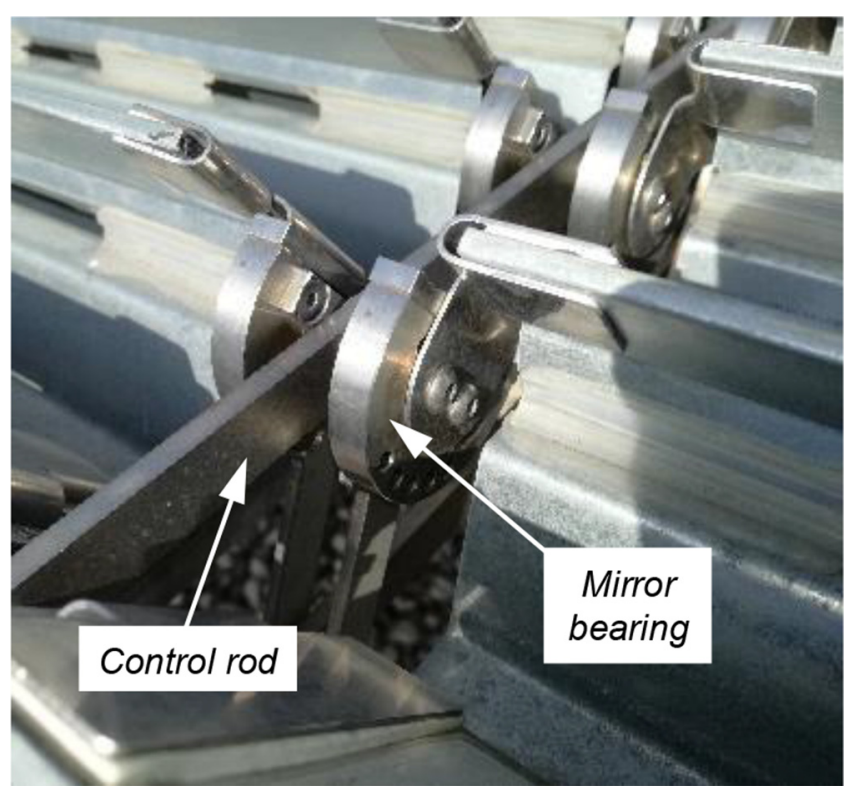

Fig. 2. Mechanical joint of control rod and mirror stripes (backwards view of mirrors).

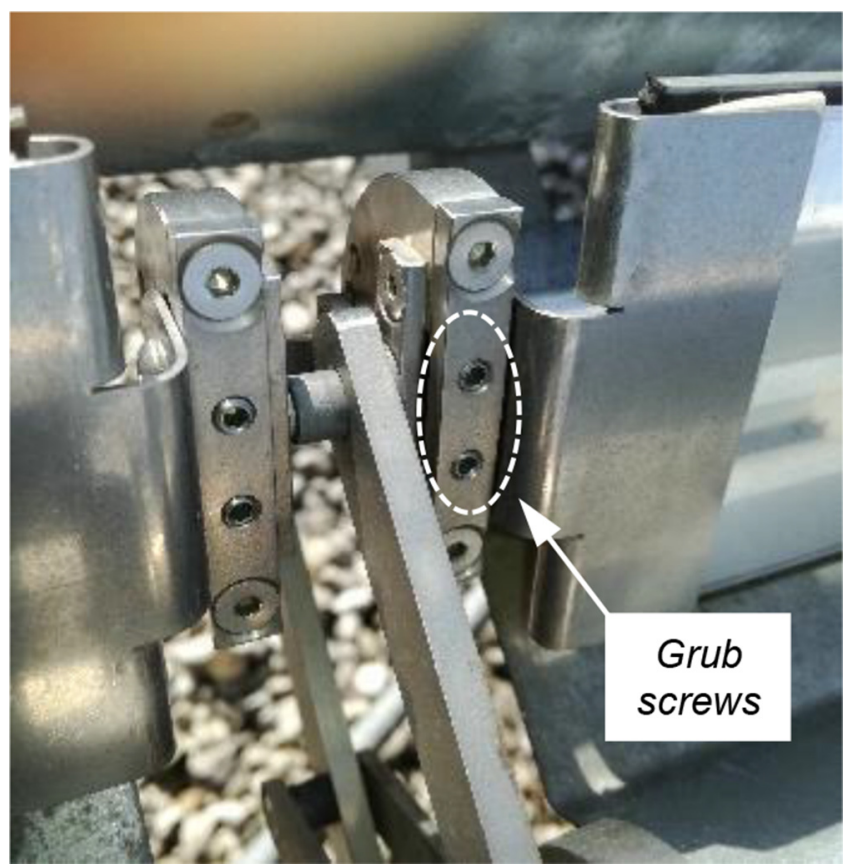

Fig. 3. Angle adjustment screws of each mirror stripe.

For the specific Fresnel collector considered in this paper, the mechanical joint between the single mirror stripes and the common control rod is done in two stages. On the one hand, the semicircular bearing of each mirror is mechanically fixed to the control rod, whereby the translational movement of the rod is transformed into a rotation of the mirror, see Figure 2. On the other hand, the mirror stripe itself is not rigidly connected to the semicircular bearing, but held in position by two grub screws on each side of its rotation axis, see Figure 3. 
These screws provide the possibility to adjust the angle of each mirror plane relative to the angle of the bearing and hence relative to the horizontal plane.

Regarding the mentioned task of re-aligning the focus from a single mirror module, the intention was to perform a geometrical calculation of each mirror stripe angle depending on the Sun position, and subsequently, to adjust the mounting angle of each mirror using a goniometer.

\subsection{Geometrical calculation of mirror stripe angles}

An entire optical 2D modelling of the Fresnel collector was performed using MATLAB ${ }^{\mathrm{TM}}$ in order to obtain the possibility to analyse optical performance parameters, e.g. resulting irradiance in the receiver input plane or concentration factor. The geometrical calculation of the single mirror stripe positions in dependence of the Sun elevation angle is an important part of this optical modelling for incorporating the internal shading mechanisms that are characteristic for a Fresnel mirror arrangement [10].

The goal of this optical modelling was to keep the model as general as possible so that it can be applied to a wide range of different Fresnel collector constructions. Therefore, the following parameters are set as inputs to the model:

- Number of mirrors.

- Width of one mirror stripe.

- Gap in between the mirror stripes and between edge mirror stripes and mirror field frame.

- Width and height of mirror field frame.

- Mounting height of mirror field frame above ground.

- Width and height of the receiver.

- Mounting height of the receiver above mirror plane.

As the receiver can have various constructive appearances, its cross section is simplified to a rectangle in the developed model. The mirror stripes are assumed to be planar without any additional bending, as this is the case in some constructions for achieving further concentration. Only the transversal fraction of the incident irradiance is taken into account for calculating the optical performance of the Fresnel mirror field at this current stage of the model, as the longitudinal portion does not cause internal mirror shading.

The geometrical calculation bases on the target that the beam reflected from the center of each mirror stripe hits the center of the receiver input plane. Hence, the required angle of each mirror stripe to the horizontal plane can be distinctly determined for varying Sun elevation angles, under consideration of the law of reflection.

Various resulting outputs can be generated by the developed optical model, e.g. the following:

- Optical efficiency: describes, which portion of the available mirror surface is illuminated and is therefore contributing to bundle irradiance on the receiver input plane.

- Resulting absolute irradiance on the receiver input plane, stepwise calculated along the width of the receiver.

- Share of each internal shading mechanism on the total shading loss.

- Mirror stripe angles to the horizontal plane.

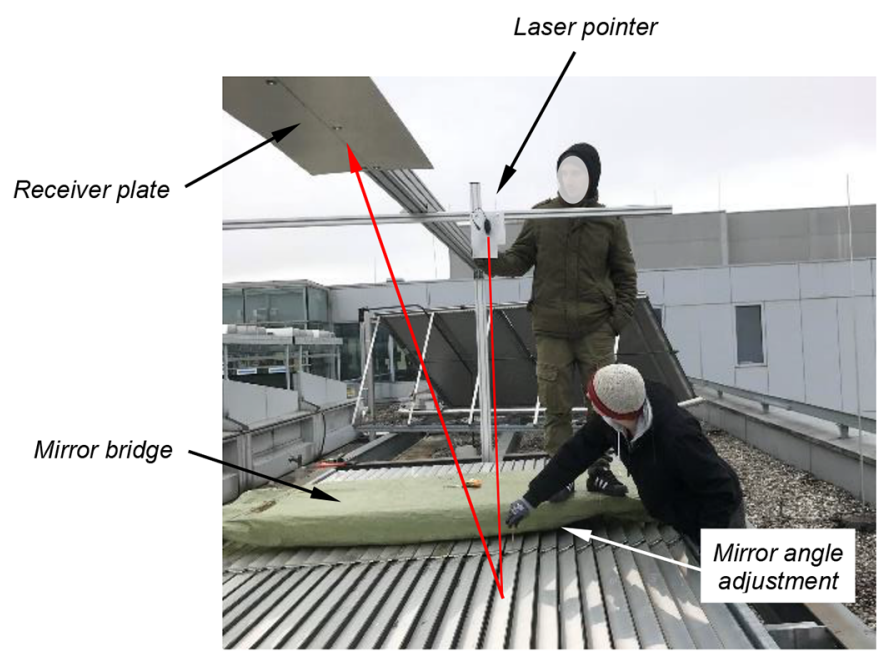

Fig. 4. Mirror alignment with laser pointer.

These mentioned model outputs are calculated in dependence of the transversal elevation angle of the Sun. Investigation of further dependencies is possible, e.g. the impact of varying the gap between the mirrors on the optical efficiency of the mirror field.

For the described task of aligning the focus on a single mirror module, the constructive parameters of the available Fresnel collector were fed into the model. The mounting height of the receiver was chosen with $1.5 \mathrm{~m}$ above the mirror plane, because this turned out to provide best optical efficiency in the collector and easy accessibility for connecting and maintaining the receiver. Subsequently, the resulting angles of the 28 mirror stripes were calculated for the case of vertical angle of incidence, with the intention to use a goniometer for adjusting the mechanical position of the mirrors, as described in the Section 2.1.

\subsection{Experimental approach to align the focus of a single mirror module}

Alternatively to the mentioned modelling approach, an experimental way for aligning the focus of a single Fresnel mirror module could be found as well, illustrated by the following Figure 4. Vertically incident Sun beams were emulated by a conventional laser pointer that was mounted in the receiver plane, pointing downwards onto the mirrors. It was adjusted accurately vertical by using a water level, and it was mounted on a horizontal aluminium profile in order to make it slidable along the width of the mirror field. Instead of the receiver, a brass plate was attached to the receiver carrying structure, so that the expected reflections from the mirrors are well visible. The assembly of a "bridge" was required in order to be able to access the mirror field without causing damages. In this configuation, the laser pointer was moved stepwise above each mirror stripe, pointing to the center of each mirror. Subsequently, each mirror angle was adjusted with the grub screws described in Section 2.1, until the reflected laser beam impinged the center of the receiver plate. 
A. Resch and T. Aigenbauer: Renew. Energy Environ. Sustain. 6, 26 (2021)

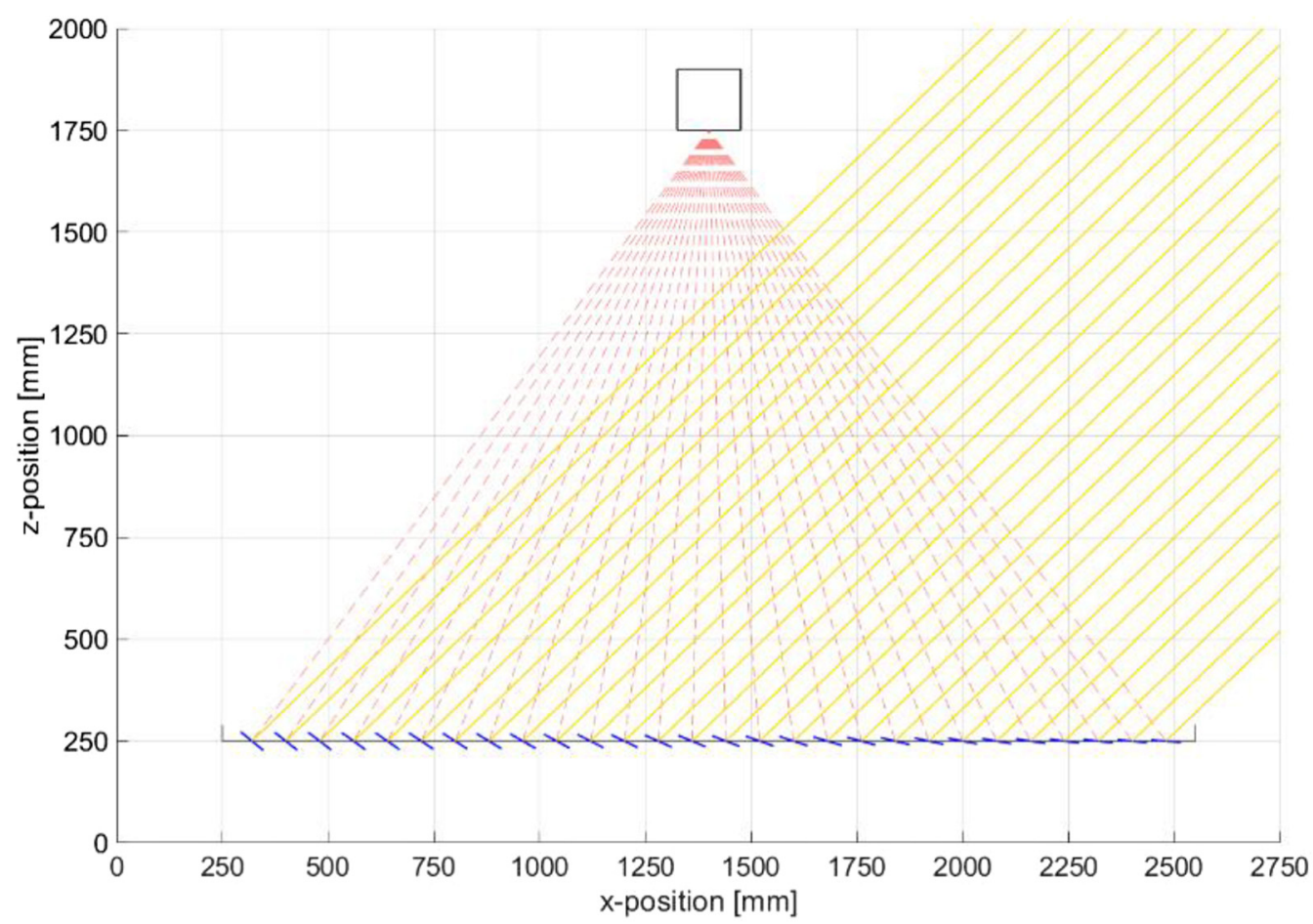

Fig. 5. 2D modelling of mirror positions at Sun elevation of $45^{\circ}$.

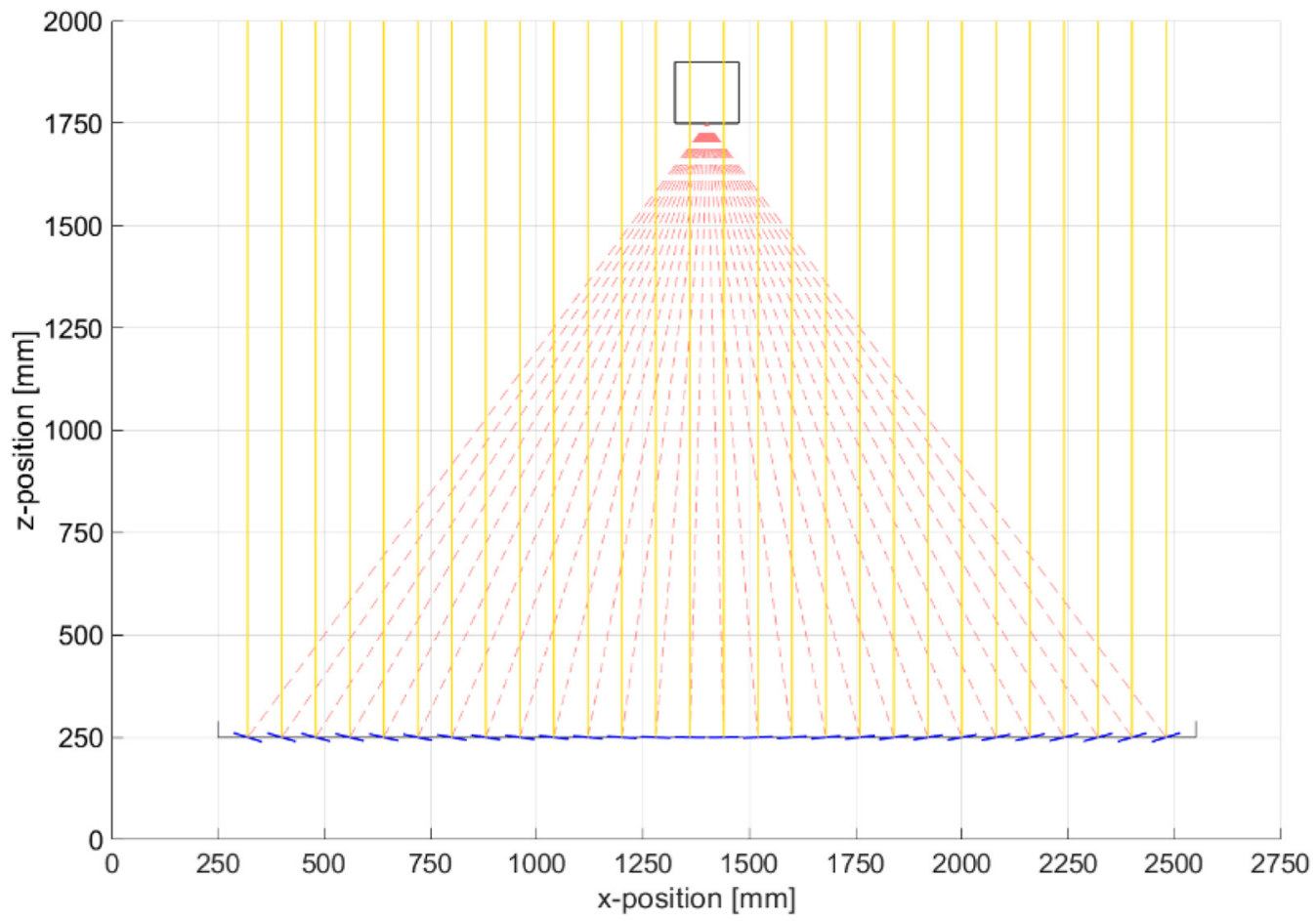

Fig. 6. 2D-modelling of mirror positions at Sun elevation of $90^{\circ}$. 
Table 1. Calculated mirror angles to horizontal plane at Sun elevation of $90^{\circ}$.

\begin{tabular}{llllllll}
\hline Mirror no. & Angle $\left[^{\circ}\right]$ & Mirror no. & Angle $\left[^{\circ}\right]$ & Mirror no. & Angle $\left[^{\circ}\right]$ & Mirror no. & Angle $\left[^{\circ}\right]$ \\
\hline 1 & 17.88 & 8 & 9.56 & 15 & -0.76 & 22 & -10.90 \\
2 & 16.84 & 9 & 8.18 & 16 & -2.29 & 23 & -12.19 \\
3 & 15.76 & 10 & 6.75 & 17 & -3.80 & 24 & -13.44 \\
4 & 14.62 & 11 & 5.29 & 18 & -5.29 & 25 & -14.62 \\
5 & 13.44 & 12 & 3.80 & 19 & -6.75 & 26 & -15.76 \\
6 & 12.19 & 13 & 2.29 & 20 & -8.18 & 27 & -16.84 \\
7 & 10.90 & 14 & 0.76 & 21 & -9.56 & 28 & -17.88 \\
\hline
\end{tabular}

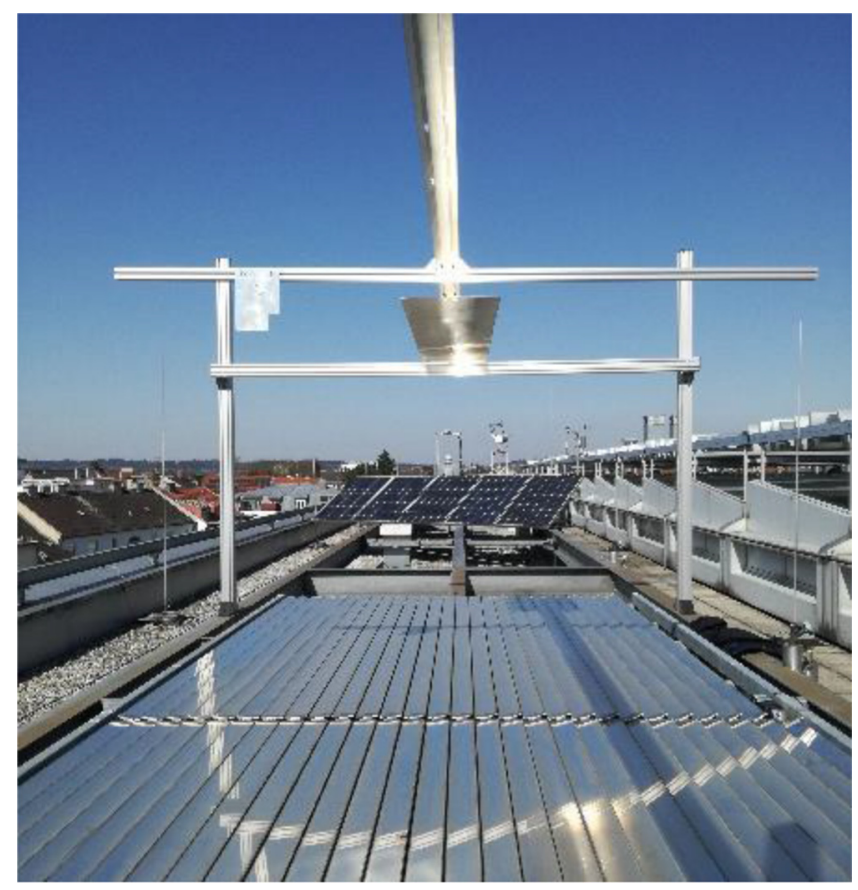

Fig. 7. Single Fresnel mirror module with receiver carrier and receiver plate after focus alignment; rooftop of FHOOE in Wels, Austria, on 26/03/2021 at 11:38 CET.

\section{Results}

The results of the chosen approaches are satisfying, as illustrated in detail within the following sub-sections.

\subsection{Results of geometrical calculation}

The results of the 2D modelling of the Fresnel collector field can be seen in the following Figure 5 (for a Sun elevation angle of $45^{\circ}$ ) and Figure 6 (for a Sun elevation angle of $90^{\circ}$ ). The indicident beams (solid yellow lines) impinge on the mirror stripes and are reflected (dashed red lines) to the center of the receiver.

The $z$-position of the mirror plane is chosen by $250 \mathrm{~mm}$, equal to the mounting height above ground. The 28 mirror stripes (in blue) reveal specific angles to the horizontal plane as expected, depending on their $x$-positions. Figure 5

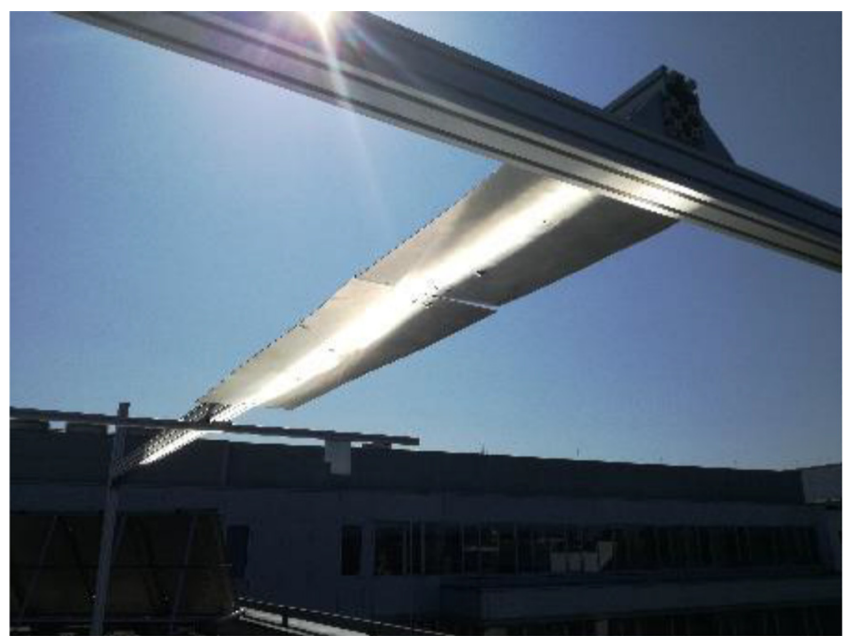

Fig. 8. Detail of focus image on the receiver plate; rooftop of FHOOE in Wels, Austria, on 26/03/2021 at 11:38 CET.

represents the resulting situation for a Sun elevation angle of $45^{\circ}$, where the different mirror angles are clearly visible, compared to Figure 6 that is calculated for an incidence angle of $90^{\circ}$.

In Figure 6, the central receiver stripes underneath the receiver appear to be close to horizontal. The exact mirror angles to the horizontal plane for this specific situation of a Sun elevation angle of $90^{\circ}$ are provided in the following Table 1.

The results of this MATLAB ${ }^{\mathrm{TM}}$ modelling has been verified by manual calculation and graphical validation that was done for randomly chosen single mirror stripes. Furthermore, the 2D model was not only checked for the present construction with 28 mirrors and the given dimensions, but all input parameters were excited within realistic boundaries in order to confirm the reliability of the developed mirror field model.

The calculated values of mirror angles from Table 1 can be used to align the focus of the available single Fresnel mirror module, if the control rod and the servo motor are in the correct position corresponding to a Sun elevation angle of $90^{\circ}$. As the angle differences between adjacent mirrors are only between $1^{\circ}$ and $1.5^{\circ}$, a precise goniometer is required in order to adjust the mirror stripes accordingly. 


\subsection{Results of experimental focus alignment}

Following the experimental approach described in Section 2.3, the 28 mirror stripes of the available single Fresnel mirror module have been aligned in such a way that the focus image appears on the receiver plate as expected, see the following Figures 7 and 8 .

Although the tracking software requires further optimiziation, the mechanical alignment of the mirrors was successful. The width of the focus image on the receiver plate as shown in Figure 8 is below $80 \mathrm{~mm}$, confirming that the single mirror images reveal an exact overlapping due to the correct mechanical adjustment of the mirror angles.

\section{Discussion}

The described procedure of adjusting the focus in a Fresnel collector system on-site due to changed mounting position of the receiver is only feasible, if the constructive design of the mirror bearing provides this possibility. In the present case, the mechanical joint between control rod and mirror stripes reveals valuable realisation with the option of adapting each mirror angle by the simple solution of adjusting two grub screws.

The theoretical approach of calculating the mirror stripe angles depending on the Sun elevation angle is promising and yields reliable results. Due to the chosen design of the optical 2D model, its application is not restricted to the specific Fresnel mirror field which is in the focus of the described work, but it is flexibly usable for different constructions of Fresnel collectors. The utilisation of the obtained mirror angle information for adjusting the mirror positions requires a digital goniometer with sufficient accuracy.

The chosen experimental way of aligning the mirror stripes according to the modified receiver position was successful. Although it requires some effort for preparing the mechanical setup, the adjusting procedure itself is rather pragmatic and simple, and the result is reliable.

\section{Conclusion}

The development of the MATLAB ${ }^{\mathrm{TM}}$ model of the Fresnel collector was done with the intention to gain an entire view on the optical performance of the system, e.g. the expected absolute irradiance on the receiver input plane. Especially, the intended flexibility of the model in terms of covering different constructions of a Fresnel collector (number of mirrors, dimensions, position of the receiver etc.) caused high effort in the modelling process. Now that the model is available, it is meaningful to use it also for realigning the focus for a single mirror module.

However, if not already available, it does not seem to be reasonable to develop an optical model from scratch, only for the considered purpose of calculating the mirror angles for focus alignment. In this case it would be recommendable to choose the demonstrated experimental way, which provides comparable reliability with significantly reduced effort.

This project is financed by research subsidies granted by the government of Upper Austria. Further thanks are dedicated to a group of students of the University of Applied Sciences Upper Austria, consisting of Tarik Cosic, Philipp Fessl, Manuel Fürnkranz, Thomas Kondler, Martin Kozole and Oliver Krofta for performing the experimental part of this research work.

\section{References}

1. REN21, Renewables 2020 Global Status Report (REN21 Secretariat, Paris, 2020)

2. RHC-Platform, Strategic Research and Innovation Agenda for Renewable Heating \& Cooling (2013)

3. W. Weiss, M. Spörk-Dür, Solar Heat Worldwide - Global Market Development and Trends in 2019 | Detailed Market Data 2018 (AEE Intec, 2020)

4. Heat Roadmap Europe, Heating and Cooling - facts and figures (2017). Available: www.heatroadmap.eu.

5. V. Quaschning, Regenerative Energiesysteme / Technologie - Berechnung - Simulation, 7th ed. (Hanser Verlag, 2011)

6. M. Kaltschmitt, W. Streicher, A. Wiese, Erneuerbare Energien / Systemtechnik, Wirtschaftlichkeit, Umweltaspekte (Springer Vieweg, 2013), vol. 5

7. C.A. Schoeneberger, C.A. McMillan, P. Kurup, S. Akar, R. Margolis, E. Masanet, Solar for industrial process heat: a review of technologies, analysis approaches, and potential applications in the United States, Energy 206, 118083 (2020)

8. A. Azouzoute et al., Thermal production and heat cost analysis of the potential of solar concentrators for industrial process applications: A case study in six sites in Morocco, Sci. African 12, e00765 (2021)

9. I. Iparraguirre et al., Solar thermal collectors for medium temperature applications: a comprehensive review and updated database, Energy Proc. 91, 64-71 (2016)

10. P. Boito, R. Grena, Application of a fixed-receiver Linear Fresnel Reflector in concentrating photovoltaics, Solar Energy 215, 198-205 (2021) 